\title{
Reflection of Plane Waves in Generalized Thermoelastic Half Space under the Action of Uniform Magnetic Field
}

\author{
Narottam Maity, ${ }^{1}$ S. P. Barik, ${ }^{2}$ and P. K. Chaudhuri ${ }^{3}$ \\ ${ }^{1}$ Department of Mathematics, Nabagram K. D. Paul Vidyalaya, 27 G. T. Road, Serampore, India \\ ${ }^{2}$ Department of Mathematics, Gobardanga Hindu College, West Bengal, India \\ ${ }^{3}$ Department of Applied Mathematics, University of Calcutta, 92 A. P. C. Road, Kolkata 700 009, India \\ Correspondence should be addressed to S. P. Barik; spbarik1@gmail.com
}

Received 27 June 2016; Accepted 5 October 2016

Academic Editor: Elio Usai

Copyright (C) 2016 Narottam Maity et al. This is an open access article distributed under the Creative Commons Attribution License, which permits unrestricted use, distribution, and reproduction in any medium, provided the original work is properly cited.

\begin{abstract}
Reflection of longitudinal displacement waves in a generalized thermoelastic half space under the action of uniform magnetic field has been investigated. The magnetic field is applied in such a direction that the problem can be considered as a two-dimensional one. The discussion is based on the three theories of generalized thermoelasticity: Lord-Shulman (L-S), Green-Lindsay (G-L), and Green-Naghdi (G-N) with energy dissipation. We compute the possible wave velocities for different models. Amplitude ratios have been presented. The effects of magnetic field on various subjects of interest are discussed and shown graphically.
\end{abstract}

\section{Introduction}

It is known that mechanical loadings are not the only cause for deformation in elastic solids; thermal loadings also can play a vital role in producing deformations in structures and machines subject to generation and flow of heat. Thermoelasticity takes care of the deformations and stresses produced due to thermal loadings along with the deformation and stresses produced due to mechanical loadings. Clearly the governing equations of motion in thermoelasticity consist of the coupled equations involving mechanical and thermal stresses and the equation governing heat flow in the solid. The parabolic type of heat conduction equation as was adopted initially in the classical theory encountered a serious drawback in the sense that the speed of the heat propagation in the solid could be infinite, which is absolutely unrealistic. Naturally, scientists endeavoured to develop a new theory compatible with the reality. In 1967 Lord and Shulman [1] employed a modified version of the Fourier law of heat conduction and developed a theory known as generalized theory of thermoelasticity. The new thermoelastic equations yielded a finite velocity of heat propagation by introducing relaxation time into the energy equation, rendering the new thermoelastic field equations fully hyperbolic. In 1972
Green and Lindsay [2] proposed a theory of thermoelasticity with certain special features that contrasts with the previous theory having a thermal relaxation time. In this theory Fourier's law of heat conduction is unchanged whereas the classical energy equation and stress-strain-temperature relations are modified. The two theories have been developed with independent ideas and one cannot be considered as a particular case of the other. Investigation of various problems characterizing the two theories has been discussed by Chandrasekharaiah $[3,4]$. Further modifications in the constitutive equations of thermoelasticity were done by Green and Naghdi [5] to accommodate a wider class of heat flow problems.

Another interesting field of recent study is the field of magnetothermoelasticity in which interacting effects of applied magnetic field on elastic and thermal deformations of a solid are studied. Such studies have applications in several areas, particularly in nuclear devices, biomedical engineering, and geomagnetic investigations. Some of the works related to the interaction of the electromagnetic field, the thermal field, and the electric field may be available in literature, namely, Abd-Alla and Al-Dawy [6, 7], Ezzat and Othman [8], Ezzat [9], Wang et al. [10], and Othman [1113]. A problem of reflection of plane waves in a rotating transversely isotropic magnetothermoelastic half space has 
been discussed by Singh and Yadav [14]. Roychoudhuri and Banerjee [15] investigated viscoelastic plane waves in a rotating thermoelastic medium following Green-Naghdi model. Thermoelastic plane waves in a rotating isotropic medium have been studied by Ahmad and Khan [16]. The effects of rotation and relaxation times on plane wave propagation in a generalized thermoelastic medium have been investigated by Roychodhuri [17].

The present paper aims at the study of reflection of longitudinal wave from the flat boundary of an elastic half space in generalized thermoelasticity. The half space is under the action of a uniform magnetic field and our objective is to study the behavior of reflection of longitudinal plane wave in presence of magnetic field. It is assumed that the direction of the magnetic field is such that the problem can be considered as two-dimensional. It is observed that there are two distinct types of longitudinal plane waves in the medium. The effects of magnetic field on the velocity of propagation of these types of waves have been shown graphically. Magnetic field effects on amplitude ratios of the waves have also been shown.

\section{Field Equations}

In our mathematical expressions we shall use bold letters to represent the vector quantities appearing in our discussion. The field equations governing the displacement $\mathbf{u}=u_{i}(\mathbf{x}, t)$ and the temperature $T(\mathbf{x}, t)$ for a homogeneous isotropic magneto elastic material in generalized magnetothermoelasticity in the absence of body force may be presented in vector form as

$$
\begin{aligned}
& (\lambda+2 \mu) \nabla(\nabla \cdot \mathbf{u})-\mu[\nabla \times(\nabla \times \mathbf{u})] \\
& \quad-\gamma\left(1+\alpha \delta_{2 k} \frac{\delta}{\delta t}\right) \nabla T+\mathbf{J} \times \mathbf{B}=\rho \ddot{\mathbf{u}} \\
& \left(\delta_{1 k}+\delta_{2 k}+\delta_{3 k} \frac{\partial}{\partial t}\right) K \nabla^{2} T+\delta_{3 k} K^{*} \nabla^{2} T \\
& =\rho C_{e}\left[\left(\delta_{1 k}+\delta_{2 k}\right) \dot{T}+\left\{\left(\delta_{1 k}+\delta_{2 k}\right) \alpha^{*}+\delta_{3 k}\right\} \ddot{T}\right] \\
& \quad+\gamma T^{*}\left[\delta_{2 k}+\delta_{3 k} \frac{\partial}{\partial t}+\delta_{1 k}\left(1+\alpha^{*} \frac{\partial}{\partial t}\right)\right] \dot{\Delta},
\end{aligned}
$$

where $\mathbf{J}$ is the current density, $\mathbf{B}$ is the magnetic induction vector, $\mathbf{B}=\mu_{0} \mathbf{H}, \mathbf{H}$ is the total magnetic field vector, $\mu_{0}$ is the magnetic permeability of solid, $\lambda, \mu$ are Lame's elastic constants, $\alpha, \alpha^{*}$ are relaxation times, $K^{*}=C_{e}(\lambda+2 \mu) / 4$ is additional material constant, $C_{e}$ is the specific heat, $T$ is the temperature, $T^{*}$ is the absolute temperature, $\alpha_{t}$ is the coefficient of linear thermal expansion, $\Delta$ is the dilatation, $\gamma=(3 \lambda+2 \mu) \alpha_{t}, K$ is the coefficient of thermal conductivity, $\rho$ is the mass density, and $\delta_{i j}$ is the Kronecker delta.

Equation (2) is a single equation representing the generalized heat conduction equation for three different models depending on the values of suffix $k$. For $k=1$, (2) represents the Lord-Shulman (L-S) model, for $k=2$ it represents the Green-Lindsay (G-L) model, and for $k=3$ it represents Green-Naghdi (G-N) model. The thermal relaxation time satisfies the inequalities [2] $\alpha \geq \alpha^{*}>0$ in the case of G-L theory.

The above field equations (1) and (2) are supplemented by the following constitutive equations:

$$
\tau_{i j}=\lambda e_{k k}+2 \mu e_{i j}-\gamma\left(T+\delta_{2 k} \alpha \dot{T}\right) \delta_{i j}
$$

in which $\tau_{i j}$ are the components of symmetric stress tensor and $e_{i j}$ are the components of strain tensor related to displacement components $u_{i}$, as

$$
e_{i j}=\frac{1}{2}\left(u_{i, j}+u_{j, i}\right) \text {, }
$$

and the indices $i, j, k$ take values 1,2 , and 3 .

Due to the application of initial magnetic field $\mathbf{H}_{0}$, an induced magnetic field $\mathbf{h}$, an induced electric field $\mathbf{E}$, and current density vector $\mathbf{J}$ are developed, so the total magnetic field $\mathbf{H}=\mathbf{H}_{0}+\mathbf{h}$. For a slowly moving homogeneous electrically conducting solid, the linear equations of electrodynamics are

$$
\begin{aligned}
\nabla \times \mathbf{h} & =\mathbf{J}+\epsilon_{0} \dot{\mathbf{E}}, \\
\nabla \times \mathbf{E} & =-\mu_{0} \dot{\mathbf{h}}, \\
\nabla \cdot \mathbf{h} & =0, \\
\mathbf{E} & =-\mu_{0}(\dot{\mathbf{u}} \times \mathbf{H}),
\end{aligned}
$$

where $\nabla=(\partial / \partial x, \partial / \partial y, \partial / \partial z), \epsilon_{0}$ is the electrical conductivity, and $\dot{\mathbf{u}}$ is the particle velocity. Here we ignore the small effect of temperature gradient on the current density vector J. The deformation vector is supposed to be small and the displacement vector is measured from the steady state deformed position.

Equation (3) when expressed in terms of displacement vector $u_{k}$ becomes

$$
\tau_{i j}=\lambda u_{k, k} \delta_{i j}+\mu\left(u_{i, j}+u_{j, i}\right)-\gamma\left(T+\delta_{2 k} \alpha \dot{T}\right) \delta_{i j} .
$$

Equation (1) may be expressed in vector notation in the form

$$
c_{1}^{2} \nabla(\nabla \cdot \mathbf{u})-c_{2}^{2} \nabla \times(\nabla \times \mathbf{u})-c_{3}^{2} \nabla T+\frac{\mu_{0}}{\rho}(\mathbf{J} \times \mathbf{H})=\ddot{\mathbf{u}},
$$

where

$$
\begin{aligned}
c_{1}^{2} & =\frac{\lambda+2 \mu}{\rho}, \\
c_{2}^{2} & =\frac{\mu}{\rho}, \\
c_{3}^{2} & =\frac{\gamma}{\rho}\left(1+\alpha \delta_{2 k} \frac{\delta}{\delta t}\right) .
\end{aligned}
$$

It is convenient to write the displacement vector $\mathbf{u}$ in terms of a scalar potential $q$ and a vector potential $\Psi$ in the form

$$
\mathbf{u}=\nabla q+\nabla \times \Psi
$$

with $\nabla \cdot \Psi=0$. 


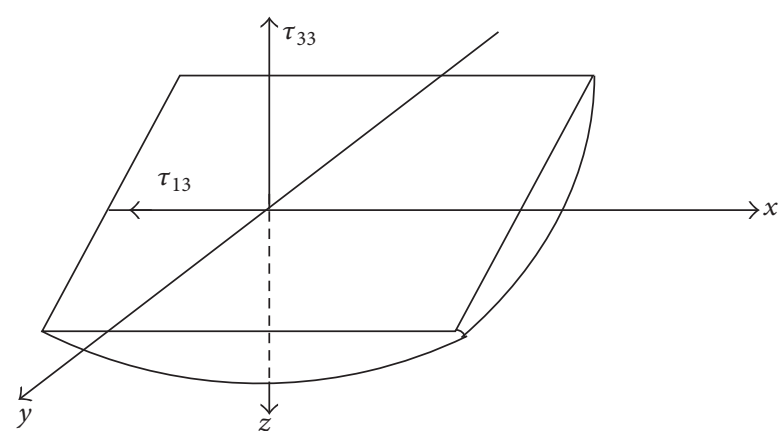

Figure 1: Geometry of the problem.

\section{Formulation of the Problem}

We take origin of Cartesian coordinate system on the free surface of the thermoelastic solid occupying a half space. $z$ axis is drawn along the normal to the free surface pointing into the medium, while $x$ and $y$ axes are taken along free surface of the half space, which is assumed to be thermally insulated (Figure 1). The solid is under the action of a uniform magnetic field $\mathbf{H}_{0}=\left(0, H_{0}, 0\right)$. The applied magnetic field will develop induced magnetic field $\mathbf{h}=(0, h, 0)$, an induced electric field $\mathbf{E}=\left(E_{1}, E_{2}, E_{3}\right)$, and current density $\mathbf{J}=$ $\left(J_{1}, 0, J_{3}\right)$.

To study the problem of reflection of a longitudinal plane wave at the free surface of the solid under thermal condition and magnetic field effect, we assume that the incident and the reflected waves are all lying on the $x-z$ plane.

Thus, for the problem under consideration the total magnetic field $\mathbf{H}=\left(0, H_{0}+h, 0\right)$ and the displacement vector $\mathbf{u}=(u, 0, w)$. Substituting (9) into (7) we obtain the following equations:

$$
\begin{aligned}
& \left(c_{1}^{2}+c_{a}^{2}\right) \nabla^{2} q-c_{1}^{2} \chi\left(1+\alpha \delta_{2 k} \frac{\delta}{\delta t}\right) T-\left(1+c_{b}\right) \ddot{q}=0, \\
& c_{2}^{2} \nabla^{2} \Psi=\left(1+c_{b}\right) \ddot{\Psi},
\end{aligned}
$$

where $c_{a}^{2}=\mu_{0} H_{0}^{2} / \rho, c_{b}=c_{a}^{2} / c_{0}^{2}, \chi=\gamma /(\lambda+2 \mu)$, $c_{0}=1 / \sqrt{\mu_{0} \epsilon_{0}}$. Clearly (10) involves both temperature and magnetic effect but (11) contains magnetic effect only. We also observe that if the medium is such that $\epsilon_{0}=0$ (i.e., no electrical conductivity), then magnetic effect is realisable in (10) only.

Substitution of (9) into (2) gives

$$
\begin{aligned}
& {\left[\left(\delta_{1 k}+\delta_{2 k}\right) K+\delta_{3 k} K^{*}\right] \nabla^{2} T+\delta_{3 k} K \nabla^{2} \dot{T}} \\
& =\rho C_{e}\left[\left(\delta_{1 k}+\delta_{2 k}\right) \dot{T}+\left\{\left(\delta_{1 k}+\delta_{2 k}\right) \alpha^{*}+\delta_{3 k}\right\} \ddot{T}\right] \\
& \quad+\gamma T^{*}\left[\delta_{2 k}+\delta_{3 k} \frac{\partial}{\partial t}+\delta_{1 k}\left(1+\alpha^{*} \frac{\partial}{\partial t}\right)\right] \nabla^{2} \dot{q} .
\end{aligned}
$$

Equations (10), (11), and (12) are three equations for the field functions $q, \Psi$, and $T$. A look at (11) reveals that the vector potential $\Psi$ is not coupled with $q$ and $T$, whereas the remaining two equations are coupled in $q$ and $T$. It is also seen that (12) does not depend on the magnetic field.

\section{Boundary Conditions}

Assuming the surface $z=0$ as stress free and considering the motion as two-dimensional in the $x-z$ plane, the boundary conditions are

$$
\tau_{33}=0=\tau_{13} \quad \text { on } z=0
$$

Also, as the boundary $z=0$ of the half space is assumed to be thermally insulated, the boundary condition may be put as

$$
\frac{\partial T}{\partial z}=0 \quad \text { on } z=0
$$

\section{Plane Wave Solution}

For the propagation of a plane wave in the medium in the direction of the unit vector $\nu$, the field functions $q, \Psi$, and $T$ may be expressed as

$$
(q, \Psi, T)=\left(q_{0}, \Psi_{0}, T_{0}\right) U(\mathbf{x}, t)
$$

where

$$
U(\mathbf{x}, t)=\operatorname{Re}\left[\exp \left(-\zeta+\frac{i \kappa}{V}\right) \boldsymbol{v} \cdot \mathbf{r} \exp (-i \kappa t)\right],
$$

$q_{0}$ and $\phi_{0}$ are constant scalars and $\Psi_{0}$ is a constant vector, $i=$ $\sqrt{-1}, \zeta(\geq 0)$ is the wave attenuation parameter, $V$ is the wave speed, and $\kappa$ is the frequency.

From (9) and (15) it follows that

$$
\begin{aligned}
\mathbf{u}= & \boldsymbol{\nu} \operatorname{Re}\left[q_{0}\left(-\zeta+\frac{i \kappa}{V}\right) \exp \left(-\zeta+\frac{i \kappa}{V}\right) \boldsymbol{\nu}\right. \\
& \cdot \mathbf{r} \exp (-i \kappa t)] .
\end{aligned}
$$

This shows that the displacement vector is in the direction of wave propagation. We shall call these waves longitudinal displacement waves.

Substituting (15) into (11) yields

$$
c_{2}^{2}\left(-\zeta+\frac{i \kappa}{V}\right)^{2}=-\kappa^{2}\left(1+c_{b}\right) .
$$

It follows from (18) that if

$$
\begin{aligned}
\zeta & =0 \\
\text { then } V^{2} & =\frac{c_{2}^{2}}{1+c_{b}} .
\end{aligned}
$$

Equation (19) shows that the speed of the transverse plane wave is affected by the presence of a magnetic field only. In case there is no magnetic field, $V=c_{2}=\sqrt{\mu / \rho}$, the classical result. 
Substituting from (15) into (10) and (12) yields

$$
\begin{aligned}
& {\left[\left(-\zeta+\frac{i \kappa}{V}\right)^{2}+\frac{\kappa^{2}}{c_{6}^{2}}\right] q_{0}-\chi^{*} T_{0}=0,} \\
& {\left[\gamma T^{*} \kappa\left(-\zeta+\frac{i \kappa}{V}\right)^{2}\left\{\left(\delta_{1 k}+\delta_{2 k}\right) i+\delta_{1 k} \alpha^{*} \kappa+\delta_{3 k} \kappa\right\}\right]} \\
& \cdot q_{0}+\left[\left(-\zeta+\frac{i \kappa}{V}\right)^{2}\right. \\
& \cdot\left\{\delta_{1 k} K+\delta_{2 k} K+\delta_{3 k}\left(i K \kappa+K^{*}\right)\right\} \\
& \left.+\rho C_{e}\left\{\left(i \kappa+\alpha^{*} \kappa^{2}\right)\left(\delta_{1 k}+\delta_{2 k}\right)+\delta_{3 k} \kappa^{2}\right\}\right] T_{0}=0 .
\end{aligned}
$$

For nonzero $q_{0}$ and $T_{0}$ the coefficient determinant of the system of (20) should be zero. This leads to

$$
\begin{aligned}
& \left(-\zeta+\frac{i \kappa}{V}\right)^{4}\left\{\delta_{1 k}+\delta_{2 k}+\delta_{3 k}\left(i K \kappa+K^{*}\right)\right\} \\
& +\left[\frac{\kappa^{2}}{c_{6}^{2}}\left\{\left(\delta_{1 k}+\delta_{2 k}\right) K+\delta_{3 k}\left(i K \kappa+K^{*}\right)\right\}\right. \\
& +\rho C_{e}\left\{\left(\delta_{1 k}+\delta_{2 k}\right)\left(i \kappa+\alpha^{*} \kappa^{2}\right)+\delta_{3 k} \kappa^{2}\right\} \\
& \left.+\gamma T^{*} \chi^{*} \kappa\left\{\delta_{1 k}\left(i+\alpha^{*} \kappa\right)+\delta_{2 k} i+\delta_{3 k} \kappa\right\}\right](-\zeta \\
& \left.+\frac{i \kappa}{V}\right)^{2}+\frac{\rho C_{e} \kappa^{2}}{c_{6}^{2}}\left(\left(\delta_{1 k}+\delta_{2 k}\right)\left(i \kappa+\alpha^{*} \kappa^{2}\right)+\delta_{3 k} \kappa^{2}\right) \\
& =0
\end{aligned}
$$

where $c_{6}^{2}=\left(c_{1}^{2}+c_{a}^{2}\right) /\left(1+c_{b}\right), \chi^{*}=\left(c_{1}^{2} \chi /\left(c_{1}^{2}+c_{a}^{2}\right)\right)\left(1-i \kappa \alpha \delta_{2 k}\right)$. From (21) it follows that both the attenuation factor $\zeta$ and the wave speed $V$ are functions of the frequency $\kappa$, which in turn means that the longitudinal wave suffers attenuation and dispersion due to the thermoelastic character of the medium. The magnetic field also influences dispersion and attenuation as is evident from the terms $c_{a}^{2}$ and $c_{b}$ present in (21).

If we drop the attenuation factor $\zeta$ and use the angular frequency $\omega$ in place of $\kappa$, for real velocity, (21) becomes

$$
a V^{4}+b V^{2}+c=0
$$

where

$$
\begin{gathered}
a=\rho C_{e}\left(\delta_{1 k}+\delta_{2 k}\right) \frac{\alpha^{*}}{K}+\frac{\delta_{3 k}}{K^{*}}, \\
b=-\left[1+\rho C_{e} c_{6}^{2}\left\{\left(\delta_{1 k}+\delta_{2 k}\right) \frac{\alpha^{*}}{K}+\frac{\delta_{3 k}}{K^{*}}\right\}\right. \\
\left.+\gamma T^{*} \chi^{*} c_{6}^{2}\left(\frac{\delta_{1 k}}{K}+\frac{\delta_{3 k}}{K^{*}}\right)\right], \\
c=c_{6}^{2} .
\end{gathered}
$$

\section{Analysis of Dispersion Relation}

It is easily observed from (22) that the wave velocity depends upon the frequency and hence longitudinal waves propagated in a magnetothermoelastic material are subject to dispersion of general wave form. Such a dispersion is also in contrast with the classical ideas. In the absence of temperature the roots of (22) become

$$
\begin{aligned}
& V_{1}^{2}=c_{6}^{2}=\frac{c_{1}^{2}+c_{a}^{2}}{1+c_{b}}, \\
& V_{2}^{2}=0 .
\end{aligned}
$$

It shows that in absence of temperature in the solid there is only one kind of velocity of propagation, which is influenced by the magnetic field.

In case there is no magnetic field, $V_{1}^{2}=c_{1}^{2}=(\lambda+2 \mu) / \rho$, the classical result.

Now the positive roots of (22) are

$$
\begin{aligned}
& V_{1}=\left(\frac{-b+\sqrt{b^{2}-4 a c}}{2 a}\right)^{1 / 2}, \\
& V_{2}=\left(\frac{-b-\sqrt{b^{2}-4 a c}}{2 a}\right)^{1 / 2} .
\end{aligned}
$$

The discriminant of (22) is

$$
\begin{aligned}
b^{2}-4 a c & =\left(1-p_{\mathrm{LS}}\right)^{2}+2\left(1+p_{\mathrm{LS}}\right) q_{\mathrm{LS}}+q_{\mathrm{LS}}^{2} \\
& \quad(\text { for L-S model }) \\
& =\left(p_{\mathrm{GL}}-1\right)^{2} \quad(\text { for G-L model }) \\
& =\left(p_{\mathrm{GN}}-1\right)^{2}+2\left(p_{\mathrm{GN}}+1\right) q_{\mathrm{GN}}+q_{\mathrm{GN}}^{2} \\
& (\text { for G-N model }),
\end{aligned}
$$

where

$$
\begin{aligned}
p_{\mathrm{LS}} & =p_{\mathrm{GL}}=\frac{\rho C_{e} \alpha^{*} c_{6}^{2}}{K}, \\
p_{\mathrm{GN}} & =\frac{\rho C_{e} c_{6}^{2}}{K^{*}}, \\
q_{\mathrm{LS}} & =\frac{\gamma \alpha^{*} \chi^{*} T^{*} c_{6}^{2}}{K}, \\
q_{\mathrm{GN}} & =\frac{\gamma \alpha^{*} \chi^{*} c_{6}^{2}}{K^{*}} .
\end{aligned}
$$

It may be easily checked that the discriminant is greater than or equal to zero.

Equation (22) yields a quadratic equation in $V^{2}$ and hence two distinct types of longitudinal waves are possible in the medium. This information is in contrast with the classical elastic material where there is only one kind of longitudinal wave propagating in the medium for all frequencies with velocity $c_{1}=((\lambda+2 \mu) / \rho)^{1 / 2}$. 
The velocity squares which are the roots of (22) for the three different considered models depend on the elastic parameters $\lambda$, $\mu$; magnetic parameters $c_{a}^{2}, c_{b}$, and temperature parameters and are given by

$$
\begin{aligned}
V_{1,2}^{2} & =\frac{K}{2 \rho C_{e}}\left[\left(1+p_{\mathrm{LS}}+q_{\mathrm{LS}}\right)\right. \\
& \left. \pm \sqrt{\left(1-p_{\mathrm{LS}}\right)^{2}+2\left(1+p_{\mathrm{LS}}\right) q_{\mathrm{LS}}+q_{\mathrm{LS}}^{2}}\right]
\end{aligned}
$$

(for L-S model)

$$
\begin{aligned}
& =c_{6}^{2}, \frac{K}{\rho C_{e}} \quad(\text { for G-L model }) \\
& =\frac{K^{*}}{2 \rho C_{e}}\left[\left(1+p_{\mathrm{GN}}+q_{\mathrm{GN}}\right)\right. \\
& \left.\quad \pm \sqrt{\left(1-p_{\mathrm{GN}}\right)^{2}+2\left(1+p_{\mathrm{GN}}\right) q_{\mathrm{GN}}+q_{\mathrm{GN}}^{2}}\right]
\end{aligned}
$$

(for G-N model) .

Here we observe that for L-S and G-N models both the velocities are coupled in temperature and magnetic field, whereas for G-L model one velocity purely depends on elastic and magnetic parameters and the other velocity depends only on temperature parameters.

\section{Reflection of Coupled Longitudinal Displacement Waves}

We suppose that a coupled longitudinal displacement wave falls on the surface $z=0$ from within the thermoelastic medium. The plane of incidence is assumed to be such that the motion lies in the $x-z$ plane only.

We suppose that the incident plane wave is advancing in the direction of the unit vector $\boldsymbol{\nu}_{1}$. After reflection at the stress free surface $z=0$, we shall have three waves moving in the directions of unit vectors $\boldsymbol{v}_{2}, \boldsymbol{v}_{3}$, and $\boldsymbol{v}_{4}$ (Figure 2) such that

(a) one set of longitudinal coupled waves propagates with velocity $V_{1}$ in the direction of $\boldsymbol{v}_{3}$,

(b) another set of longitudinal coupled waves propagates with velocity $V_{2}$ in the direction of $\boldsymbol{v}_{2}$,

(c) a third set of transverse waves propagates with velocity $V_{3}$ in the direction of $\boldsymbol{v}_{4}$.

For the incident wave the scalar potential $q_{1}$ and temperature $T_{1}$ are expressed in terms of the circular frequency $\omega_{1}$ and wave number $k_{1}$ in the form

$$
\begin{aligned}
& q_{1}=a_{1} \exp \left[i\left(k_{1} \boldsymbol{v}_{1} \cdot \mathbf{r}-\omega_{1} t\right)\right], \\
& T_{1}=b_{1} \exp \left[i\left(k_{1} \boldsymbol{\nu}_{1} \cdot \mathbf{r}-\omega_{1} t\right)\right] .
\end{aligned}
$$

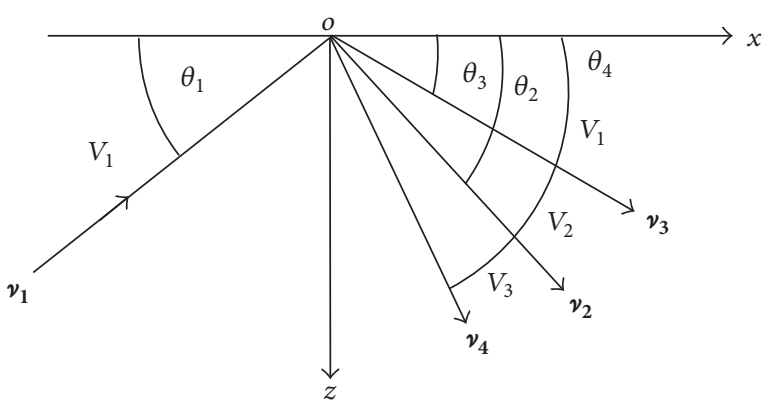

FIgURE 2: Reflection of longitudinal displacement wave.

Because there will be three types of reflected waves, namely, two distinct longitudinal and one transverse, the corresponding potentials $q_{2}, q_{3}$, temperature parameters $T_{2}, T_{3}$, and $\Psi$ are represented as

$$
\begin{aligned}
& q_{2}=a_{2} \exp \left[i\left(k_{2} \boldsymbol{v}_{2} \cdot \mathbf{r}-\omega_{2} t\right)\right], \\
& T_{2}=b_{2} \exp \left[i\left(k_{2} \boldsymbol{v}_{2} \cdot \mathbf{r}-\omega_{2} t\right)\right], \\
& q_{3}=a_{3} \exp \left[i\left(k_{1} \boldsymbol{v}_{3} \cdot \mathbf{r}-\omega_{1} t\right)\right], \\
& T_{3}=b_{3} \exp \left[i\left(k_{1} \boldsymbol{v}_{3} \cdot \mathbf{r}-\omega_{1} t\right)\right], \\
& \Psi=\boldsymbol{\eta} A \exp \left[i\left(k_{3} \boldsymbol{v}_{4} \cdot \mathbf{r}-\omega_{3} t\right)\right],
\end{aligned}
$$

where $\omega_{1}=k_{1} V_{1}, \omega_{2}=k_{2} V_{2}, \omega_{3}=k_{3} V_{3}$, and $\boldsymbol{\eta}$ is a unit vector along $y$-axis.

\section{Basic Solutions}

We shall use relations (3) and (9) in the boundary condition (13) to get

$$
\begin{aligned}
2 c_{2}^{2}\left(\Psi_{, x z}-q_{, x x}\right)+\left(1+c_{b}\right) q_{, t t}-c_{a}^{2}\left(q_{, x x}+q_{, z z}\right) & =0 \\
\text { at } z & =0, \\
2 q_{, x z}+\Psi_{, x x}-\Psi_{, z z} & =0 \\
\text { at } z & =0 .
\end{aligned}
$$

Using (10) we get

$$
T=\frac{c_{1}^{2}+c_{a}^{2}}{c_{3}^{2}}\left(q_{, x x}+q_{, z z}\right)-\frac{1+c_{b}}{c_{3}^{2}} q_{, t t},
$$

where $q=q_{1}+q_{2}+q_{3}$.

The solution of the reflection problem includes the determination of the amplitude and directions of the reflected waves corresponding to an incident wave. Using the potential representation (29) we find that the stress boundary conditions are satisfied at $z=0$ if

$$
\begin{gathered}
\omega_{1}=\omega_{2}=\omega_{3}, \\
k_{1} v_{1 x}=k_{2} v_{2 x}=k_{1} v_{3 x}=k_{3} v_{4 x}, \\
k_{1} v_{1 y}=k_{2} \nu_{2 y}=k_{1} v_{3 y}=k_{3} v_{4 y} .
\end{gathered}
$$


Since the incident wave is in the $x-z$ plane, $v_{1 y}=0$ we have from (35)

$$
v_{2 y}=v_{3 y}=v_{4 y}=0 \text {. }
$$

Now use of the boundary conditions (13), (14), (31), and (32) yields

$$
\begin{array}{r}
m_{1} a_{1}+m_{2} a_{2}+m_{3} a_{3}-m_{4} A=0, \\
m_{1}^{\prime} a_{1}+m_{2}^{\prime} a_{2}+m_{3}^{\prime} a_{3}+m_{4}^{\prime} A=0, \\
m_{1}^{\prime \prime} a_{1}+m_{2}^{\prime \prime} a_{2}+m_{3}^{\prime \prime} a_{3}=0,
\end{array}
$$

where

$$
\begin{aligned}
& m_{1}=2 c_{2}^{2} v_{1 x}^{2}-\left(1+c_{b}\right) \frac{\omega_{1}^{2}}{k_{1}^{2}}+c_{a}^{2}, \\
& m_{2}=2 c_{2}^{2} v_{1 x}^{2}-\left(1+c_{b}\right) \frac{\omega_{2}^{2}}{k_{1}^{2}}+c_{a}^{2} \\
& m_{3}=2 c_{2}^{2} v_{3 x}^{2}-\left(1+c_{b}\right) \frac{\omega_{1}^{2}}{k_{1}^{2}}+c_{a}^{2} \\
& m_{4}=2 c_{2}^{2} v_{1 x}^{2} \frac{v_{4 z}}{v_{4 x}} \\
& m_{1}^{\prime}=2 v_{1 x} v_{1 z}, \\
& m_{2}^{\prime}=2 v_{1 x}^{2} \frac{v_{2 z}}{v_{2 x}}, \\
& m_{3}^{\prime}=2 v_{3 x} v_{3 z}, \\
& m_{3}^{\prime \prime}=\frac{\omega_{1}^{2}}{k_{1}^{2}} v_{3 z}^{2}-c_{6}^{2} v_{3 z} . \\
& m_{1}^{\prime \prime}=\frac{\omega_{2}^{2}}{k_{1}^{2}} \frac{\omega_{1 x}^{2}}{v_{1 x}^{2}}, \\
& m_{1 z}^{2}-c_{6 x}^{2} v_{1 z}
\end{aligned}
$$

Again from (34) we get

$$
\frac{v_{1 x}}{V_{1}}=\frac{v_{2 x}}{V_{2}}=\frac{v_{3 x}}{V_{1}}=\frac{v_{4 x}}{V_{3}} .
$$

Let $\theta_{i}$ be the angle made by the incident wave $(i=1)$ and three reflected waves $(i=2,3,4)$ with the normal to the half space (Figure 2). Equation (39) implies that

$$
\begin{aligned}
& \cos \theta_{3}=\cos \theta_{1}, \\
& \cos \theta_{2}=\frac{V_{2}}{V_{1}} \cos \theta_{1}, \\
& \cos \theta_{4}=\frac{V_{3}}{V_{1}} \cos \theta_{1},
\end{aligned}
$$

from which we get $\theta_{3}=\theta_{1}$.
Solving (37) we obtain the amplitude ratios in the following form:

$$
\begin{aligned}
\frac{a_{2}}{a_{1}}= & {\left[2 Q_{1}^{2} \tan \theta_{1}\left(1-\tan ^{2} \theta_{4}\right)\left(Q_{2}^{2}-Q_{2}^{\prime 2} \tan ^{2} \theta_{1}\right)\right] } \\
\cdot & {\left[\left(1-\tan ^{2} \theta_{4}\right)\left(Q_{2}^{2}-Q_{2}^{\prime 2} \tan ^{2} \theta_{1}\right)\right.} \\
\cdot & \left(Q_{3}^{2} \tan \theta_{2}-Q_{1}^{2} \tan \theta_{1}\right)+Q_{4}^{2}\left(Q_{3}^{2}-Q_{1}^{2}\right) \tan \theta_{1} \\
\cdot & \left.\tan \theta_{2} \tan \theta_{4}\right]^{-1},
\end{aligned}
$$$$
\frac{a_{3}}{a_{1}}=\left[\left(Q_{2}^{2}-Q_{2}^{\prime 2} \tan ^{2} \theta_{1}\right)\left(1-\tan ^{2} \theta_{4}\right)\right.
$$$$
\cdot\left(-Q_{1}^{2} \tan \theta_{1}-Q_{3}^{2} \tan \theta_{2}\right)+Q_{5}^{2} Q_{7}^{2} \tan \theta_{1} \tan \theta_{2}
$$$$
\left.\cdot \tan \theta_{4}\right]\left[\left(1-\tan ^{2} \theta_{4}\right)\left(Q_{2}^{2}-Q_{2}^{\prime 2} \tan ^{2} \theta_{1}\right)\right.
$$$$
\text { - }\left(Q_{3}^{2} \tan \theta_{2}-Q_{1}^{2} \tan \theta_{1}\right)+Q_{4}^{2}\left(Q_{3}^{2}-Q_{1}^{2}\right) \tan \theta_{1}
$$$$
\left.\cdot \tan \theta_{2} \tan \theta_{4}\right]^{-1}
$$$$
\frac{A}{a_{1}}=\left[Q_{5}^{2} Q_{6}^{2} \tan \theta_{1} \tan \theta_{2}\left(Q_{2}^{2}-Q_{2}^{\prime 2} \tan ^{2} \theta_{1}\right)\right]
$$$$
\cdot\left[\left(1-\tan ^{2} \theta_{4}\right)\left(Q_{2}^{2}-Q_{2}^{\prime 2} \tan ^{2} \theta_{1}\right)\right.
$$$$
\text { - }\left(Q_{3}^{2} \tan \theta_{2}-Q_{1}^{2} \tan \theta_{1}\right)+Q_{4}^{2}\left(Q_{3}^{2}-Q_{1}^{2}\right) \tan \theta_{1}
$$$$
\left.\cdot \tan \theta_{2} \tan \theta_{4}\right]^{-1}
$$

where

$$
\begin{aligned}
& Q_{1}^{2}=\frac{\omega^{2}}{k_{1}^{2}}-c_{6}^{2}, \\
& Q_{2}^{2}=\frac{2 c_{2}^{2}}{\omega^{2} / k_{1}^{2}}-Q_{2}^{\prime 2}, \\
& Q_{2}^{\prime 2}=\left(1+c_{b}\right) \frac{\omega^{2}}{k_{1}^{2}}-\frac{c_{a}^{2}}{\omega^{2} / k_{1}^{2}}, \\
& Q_{3}^{2}=\frac{\omega^{2}}{k_{1}^{2}}-c_{6}^{2} \frac{V_{1}^{2}}{V_{2}^{2}}, \\
& Q_{4}^{2}=\frac{4 c_{2}^{2}}{\omega^{2} / k_{1}^{2}}, \\
& Q_{5}^{2}=1-\frac{V_{1}^{2}}{V_{2}^{2}}, \\
& Q_{6}^{2}=4 c_{6}^{2}, \\
& Q_{7}^{2}=\frac{4 c_{2}^{2} c_{6}^{2}}{\omega^{2} / k_{1}^{2}},
\end{aligned}
$$

in which $\omega$ is the common value of $\omega_{1}, \omega_{2}, \omega_{3}$. 


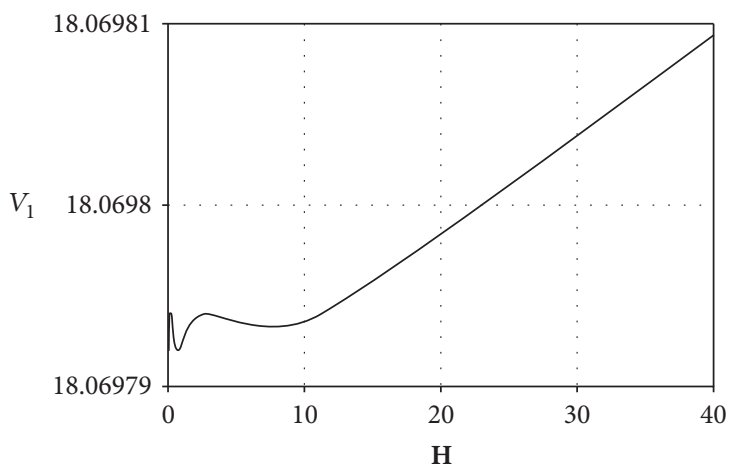

(a)

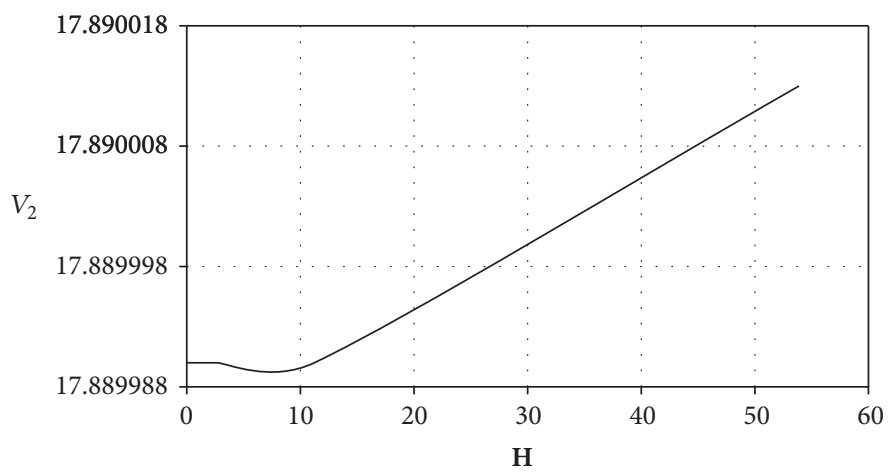

(b)

FIGURE 3: (a) Variation of possible wave velocity $V_{1}$ with $\mathbf{H}$ for L-S model. (b) Variation of possible wave velocity $V_{2}$ with $\mathbf{H}$ for L-S model.

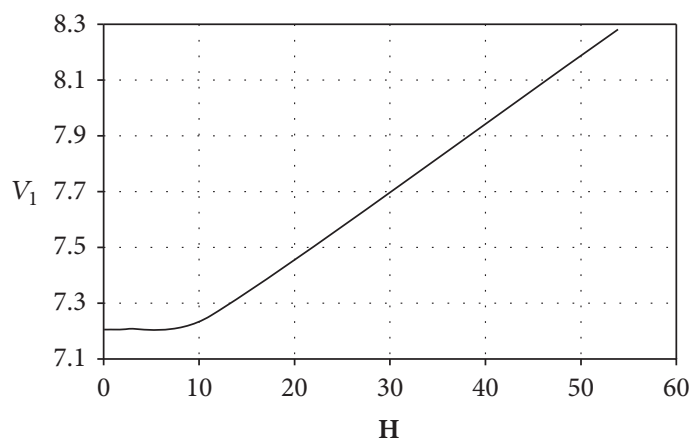

(a)

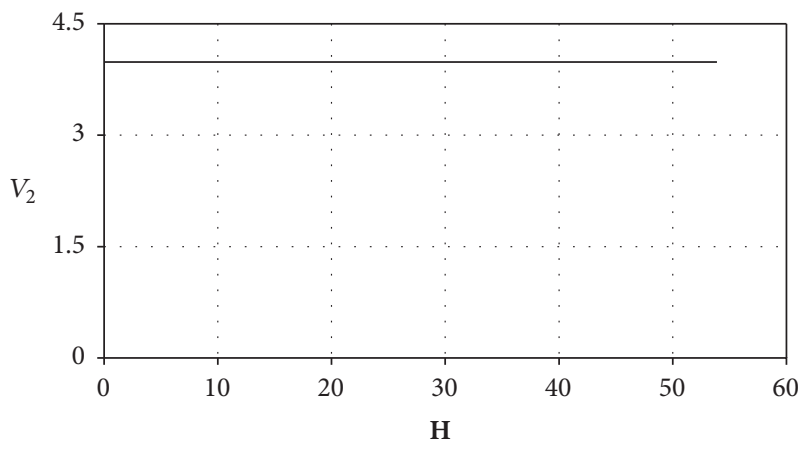

(b)

Figure 4: (a) Variation of possible wave velocity $V_{1}$ with $\mathbf{H}$ for G-L model. (b) Variation of possible wave velocity $V_{2}$ with $\mathbf{H}$ for G-L model.

\section{Numerical Results and Discussions}

The present study investigates the effects of magnetic field on the reflection problem of longitudinal waves in a thermoelastic medium. In our numerical computation we have considered aluminium-epoxy like material to illustrate theoretical results. In the present discussion we have taken the values of elastic and thermal parameters (Sharma et al. [18, 19]), as

$$
\begin{aligned}
\rho & =2.19 \mathrm{gm} / \mathrm{cm}^{3}, \\
T^{*} & =20^{\circ} \mathrm{C}, \\
\lambda & =7.59 \times 10^{11} \mathrm{dyne} / \mathrm{cm}^{2}, \\
\mu & =1.89 \times 10^{11} \mathrm{dyne} / \mathrm{cm}^{2}, \\
C_{e} & =0.23 \mathrm{cal} / \mathrm{gm}^{\circ} \mathrm{C}, \\
K & =0.6 \times 10^{-2} \mathrm{cal} / \mathrm{cm}^{\circ} \mathrm{C}, \\
\alpha^{*} & =0.5 \times 10^{-13} \mathrm{sec}, \\
\alpha & =0.75 \times 10^{-13} \mathrm{sec}, \\
\alpha_{t} & =8.1 \times 10^{-6} \mathrm{~K}^{-1}, \\
\epsilon & =0.073 .
\end{aligned}
$$

The longitudinal wave velocities $V_{1}$ and $V_{2}$ of first and second kind in magnetothermoelastic medium for three different models have been computed numerically based on the above data and have been graphically presented in Figures 3-5. The variations of velocity $V_{1}$ with applied magnetic field magnitudes in L-S, G-L, and G-N models are shown in Figures 3(a), 4(a), and 5(a) respectively, while the variations of $V_{2}$ with applied magnetic field magnitudes are presented in Figures 3(b), 4(b), and 5(b). The behaviors of $V_{1}$ in three different models are different. In L-S model, for a low magnetic field, $V_{1}$ has some oscillatory character, after which, with the increase of magnetic field, the velocity shows a steady increase. In G-L model, under low magnetic effect, the velocity appears constant, but, with the increase of field magnitude, it increases steadily. The behavior of $V_{1}$ in G-N model is showing some different character. With the increase of field magnitude from zero value, it shows constant value first, then $V_{1}$ drops down, and thereafter it increases steadily. The value of $V_{2}$ remains constant for some low value of the applied field, then it slightly drops down, and thereafter it steadily increases in L-S model. In G-L model $V_{2}$ remains unaffected by applied magnetic field. In G-L model, the behavior shows that $V_{2}$ remains unaffected for some low value of applied field but it increases steadily with higher values.

Figures 6,7 , and 8 show the variation of amplitude ratios $a_{2} / a_{1}, a_{3} / a_{1}$, and $A / a_{1}$ with respect to $\theta_{1}$ for different 


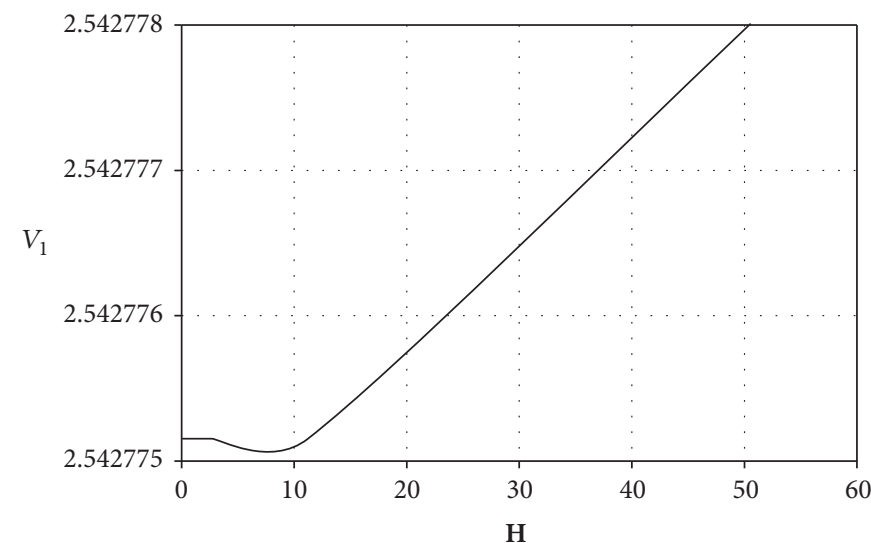

(a)

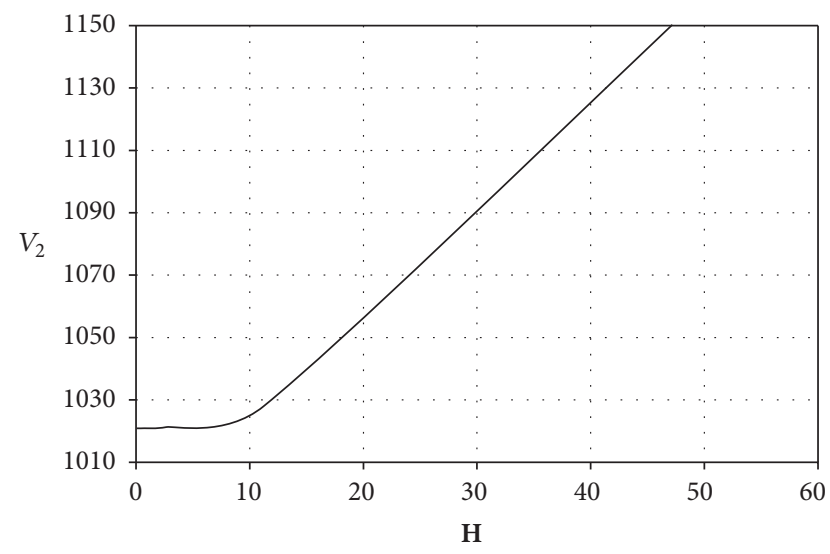

(b)

Figure 5: (a) Variation of possible wave velocity $V_{1}$ with $\mathbf{H}$ for G-N model. (b) Variation of possible wave velocity $V_{2}$ with $\mathbf{H}$ for G-N model.

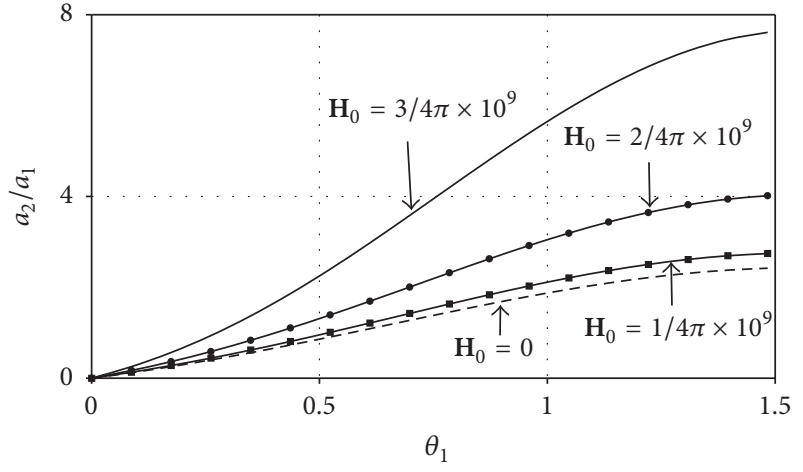

FIgURE 6: Effect of magnetic field on amplitude ratio $a_{2} / a_{1}$.

magnetic fields. Figure 6 illustrates the effect of magnetic field on the amplitude ratio $a_{2} / a_{1}$. It is observed that continuous increase of $\theta_{1}\left(0 \leq \theta_{1}<90^{\circ}\right)$ corresponds to a continuous increase of $a_{2} / a_{1}$, in absence or presence of the magnetic field. The graphs show that the values of amplitude ratio $a_{2} / a_{1}$ for the presence of magnetic field are always greater than those in absence of the magnetic field. An important observation can be made from Figure 6 that for a particular value of $\theta_{1}$ the effects of magnetic field on the amplitude ratio $a_{2} / a_{1}$ increase as magnetic field increases.

The variations of the amplitude ratio $a_{3} / a_{1}$ with respect to $\theta_{1}$ are shown in Figure 7 . It is clear from the figure that all the values of $a_{3} / a_{1}$ are negative. The absolute value of $a_{3} / a_{1}$ at $\theta_{1}=0$ is nonzero. It is observed that the absolute values of amplitude ratio $a_{3} / a_{1}$ for the presence of magnetic field are always greater than those in absence of magnetic field. It is also noted that for a particular value of $\theta_{1}$ the differences between the absolute values of amplitude ratios $a_{3} / a_{1}$ increase as magnetic field increases.

Figure 8 depicts the variation of the amplitude ratio $A / a_{1}$ with $\theta_{1}$. It is observed that, for all values of magnetic field, $A / a_{1}$ increases and attains its maximum value in the neighbourhood of $\theta_{1}=50^{\circ}$ approximately. Beyond this value of $\theta_{1}, A / a_{1}$ decreases. The nature $A / a_{1}$ is almost symmetrical

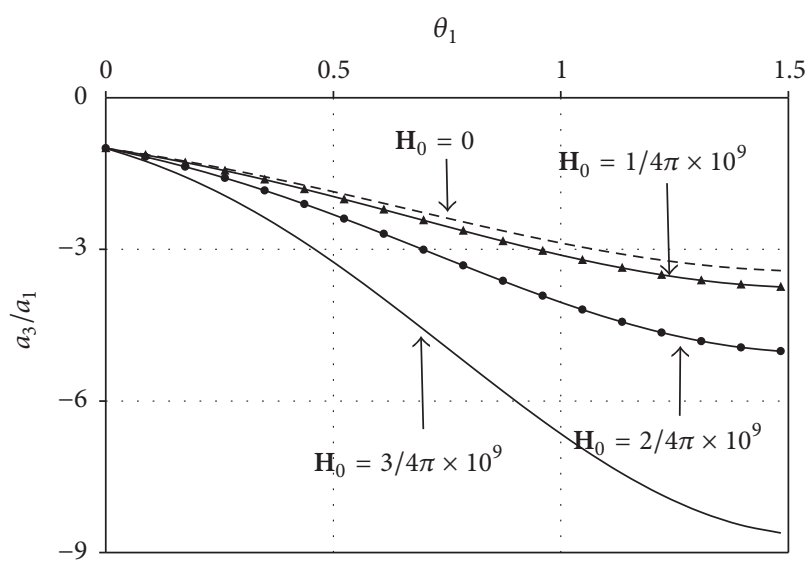

Figure 7: Effect of magnetic field on amplitude ratio $a_{3} / a_{1}$.

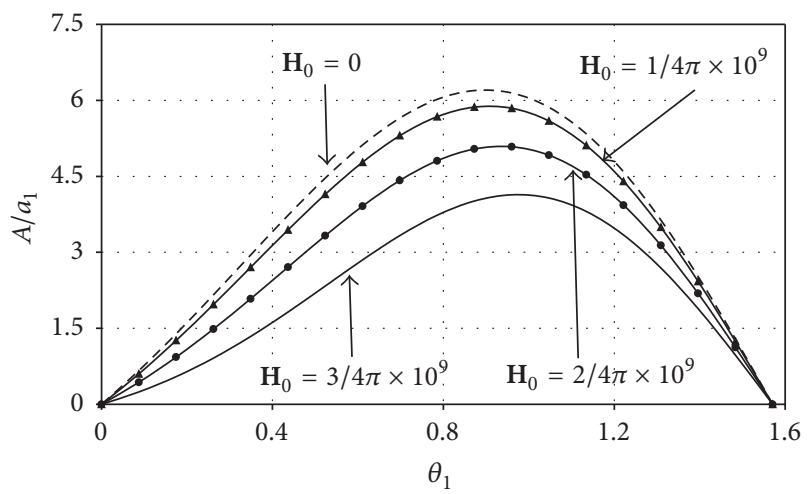

Figure 8: Effect of magnetic field on the amplitude ratio $A / a_{1}$.

around $\theta_{1}=50^{\circ}$ in $0<\theta_{1}<90^{\circ}$ for all magnitudes of applied magnetic field. The values of amplitude ratio $A / a_{1}$ for the presence of magnetic field are always less than those in absence of magnetic field. For a particular value of $\theta_{1}$ the decrement of $A / a_{1}$ is observed as magnetic field increases.

An important observation of our discussed problem due to the presence of temperature is shown in Table 1. Here, 
TABLE 1: Values of velocity $V_{1}$ in absence and presence of temperature for different models.

\begin{tabular}{lcccc}
\hline $\mathbf{H}$ & $V_{0}$ & $V_{\mathrm{LS}}$ & $V_{\mathrm{GL}}$ & $V_{\mathrm{GN}}$ \\
\hline 0.0477 & 7.2054019 & 18.069792 & 7.2054019 & 2.542775154 \\
0.0573 & 7.2054024 & 18.069792 & 7.2054024 & 2.542775154 \\
0.1031 & 7.2054057 & 18.069794 & 7.2054057 & 2.542775154 \\
0.2475 & 7.2054057 & 18.069794 & 7.2054057 & 2.542775154 \\
0.7425 & 7.2056208 & 18.069792 & 7.2056208 & 2.542775154 \\
2.6732 & 7.2082462 & 18.069794 & 7.2082462 & 2.542775154 \\
11.2274 & 7.2554202 & 18.069794 & 7.2554202 & 2.542775154 \\
53.8916 & 8.2814789 & 18.069817 & 8.2814789 & 2.542778254 \\
\hline
\end{tabular}

$V_{0}$ represents the one type of velocity values in absence of temperature and $V_{\mathrm{LS}}, V_{\mathrm{GL}}, V_{\mathrm{GN}}$ represent the respective velocities for Lord-Shulman, Green-Lindsay, and GreenNaghdi models.

\section{Conclusion}

The study focuses on how a plane longitudinal wave propagating in a generalized thermoelastic medium is affected by an applied magnetic field. The velocity of plane longitudinal waves depends heavily on the thermoelastic character of the medium as well as on the magnetic field. The velocity of the waves also varies in three different generalized thermoelastic models and the nature of the velocities in L-S, G-L, and G$\mathrm{N}$ models is significantly different, particularly at low values of the applied magnetic field. For higher values of applied magnetic field, the nature of the velocities in these models is almost similar.

\section{Competing Interests}

The authors declare that they have no competing interests.

\section{References}

[1] H. W. Lord and Y. Shulman, "A generalized dynamical theory of thermoelasticity," Journal of the Mechanics and Physics of Solids, vol. 15, no. 5, pp. 299-309, 1967.

[2] A. E. Green and K. A. Lindsay, "Thermoelasticity," Journal of Elasticity, vol. 2, no. 1, pp. 1-7, 1972.

[3] D. S. Chandrasekharaiah, "Thermoelasticity with second sound: a review," Applied Mechanics Reviews, vol. 39, no. 3, pp. 355-376, 1986.

[4] D. S. Chandrasekharaiah, "Hyperbolic thermoelasticity: a review of recent literature," Applied Mechanics Reviews, vol. 51, no. 12 , pp. 705-729, 1998.

[5] A. E. Green and P. M. Naghdi, "Thermoelasticity without energy dissipation," Journal of Elasticity, vol. 31, no. 3, pp. 189-208, 1993.

[6] A. N. Abd-Alla and A. A. Al-Dawy, "The reflection phenomena of SV-waves in a generalized thermoelastic medium," International Journal of Mathematics and Mathematical Sciences, vol. 23, no. 8, pp. 529-546, 2000.

[7] A.-E. N. Abd-Alla, "Relaxation effects on reflection of generalized magneto-thermoelastic waves," Mechanics Research Communications, vol. 27, no. 5, pp. 591-600, 2000.
[8] M. A. Ezzat and M. I. A. Othman, "Electromagnetothermoelastic plane waves with two relaxation times in a medium of perfect conductivity," International Journal of Engineering Science, vol. 38, no. 1, pp. 107-120, 2000.

[9] M. A. Ezzat, "Fundamental solution in generalized magnetothermoelasticity with two relaxation times for perfect conductor cylindrical region," International Journal of Engineering Science, vol. 42, no. 13-14, pp. 1503-1519, 2004.

[10] X. Wang, G. Lu, and S. R. Guillow, "Magnetothermodynamic stress and perturbation of magnetic field vector in a solid cylinder," Journal of Thermal Stresses, vol. 25, no. 10, pp. 909926, 2002.

[11] M. I. A. Othman and Y. Song, "The effect of rotation on the reflection of magneto-thermoelastic waves under thermoelasticity without energy dissipation," Acta Mechanica, vol. 184, no. 1-4, pp. 189-204, 2006.

[12] M. I. A. Othman, "Relaxation effects on thermal shock problems in an elastic half-space of generalized magneto-thermoelastic waves," Mechanics and Mechanical Engineering, vol. 7, no. 2, pp. 165-178, 2004.

[13] M. I. A. Othman, "Generalized thermoelasticity plane waves in rotating media with thermal relaxation under the temperature dependent properties," Mechanics and Mechanical Engineering, vol. 9, no. 2, pp. 89-100, 2005.

[14] B. Singh and A. K. Yadav, "Reflection of plane waves in a rotating transversely isotropic magneto-thermoelastic solid half-space," Journal of Theoretical and Applied Mechanics, vol. 42, no. 3, pp. 33-60, 2012.

[15] S. K. Roychoudhuri and M. Banerjee, "Magnetoelastic plane waves in rotating media in thermoelasticity of type II (G-N model)," International Journal of Mathematics and Mathematical Sciences, vol. 71, pp. 3917-3929, 2004.

[16] F. Ahmad and A. Khan, "Thermoelastic plane waves in a rotating isotropic medium," Acta Mechanica, vol. 136, no. 3, pp. 243-247, 1999.

[17] S. K. Roychodhuri, "Effect of rotation and relaxation times on plane waves in generalizedthermoelasticity," Journal of Elasticity, vol. 15, no. 1, pp. 59-68, 1985.

[18] J. N. Sharma, R. S. Chauhan, and R. Kumar, "Time-harmonic sources in a generalized thermoelastic continuum," Journal of Thermal Stresses, vol. 23, no. 7, pp. 657-674, 2000.

[19] J. N. Sharma, V. Kumar, and D. Chand, "Reflection of generalized thermoelastic waves from the boundary of a half-space," Journal of Thermal Stresses, vol. 26, no. 10, pp. 925-942, 2003. 


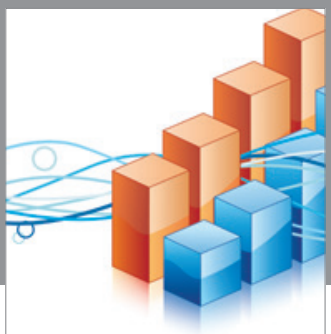

Advances in

Operations Research

vatem alat4

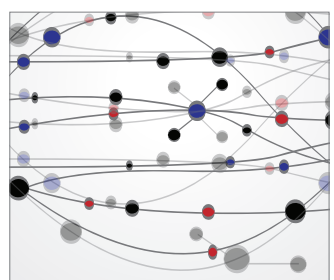

\section{The Scientific} World Journal
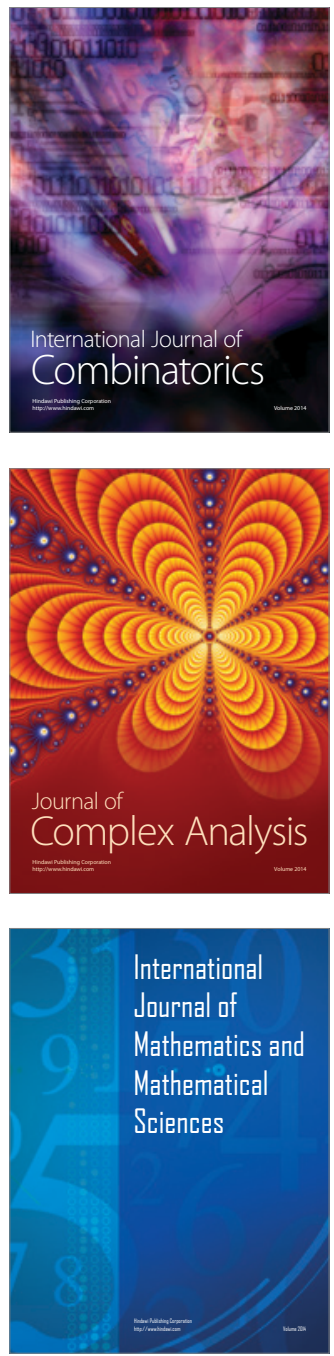
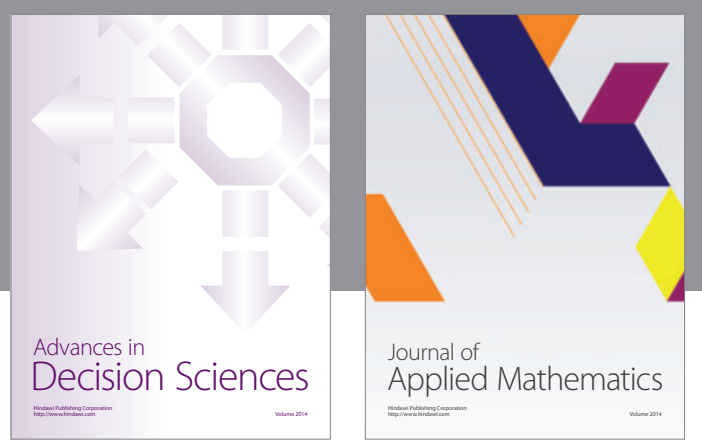

Algebra

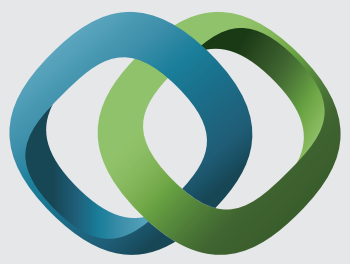

\section{Hindawi}

Submit your manuscripts at

http://www.hindawi.com
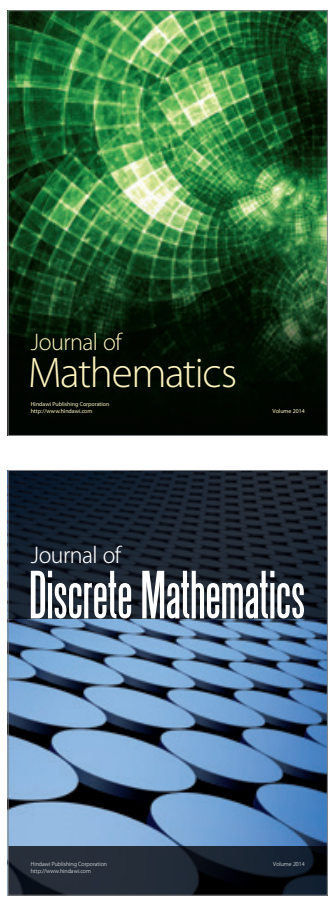

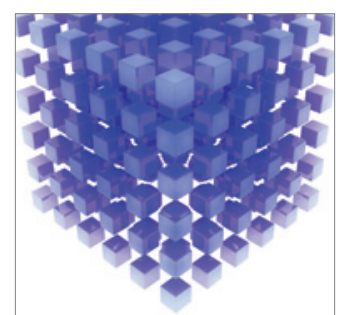

Mathematical Problems in Engineering
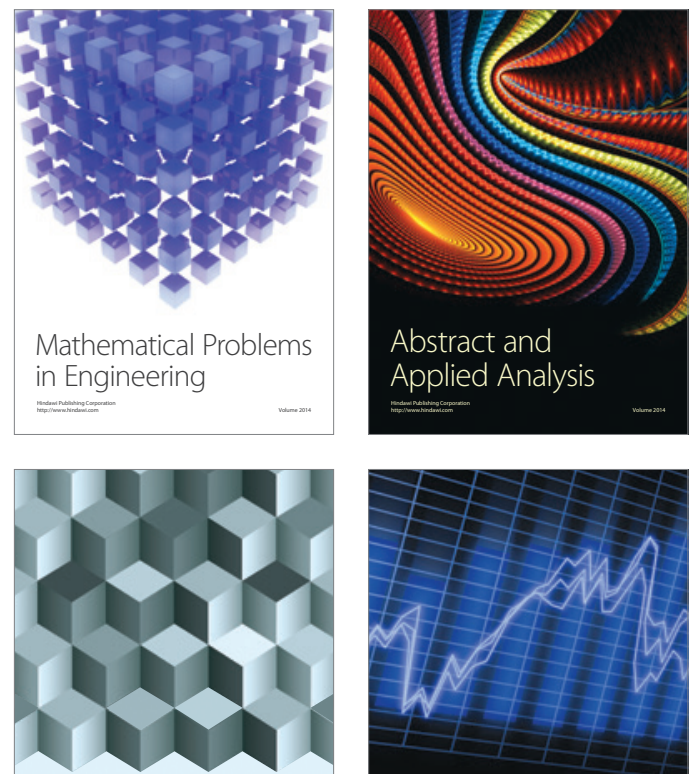

Journal of

Function Spaces

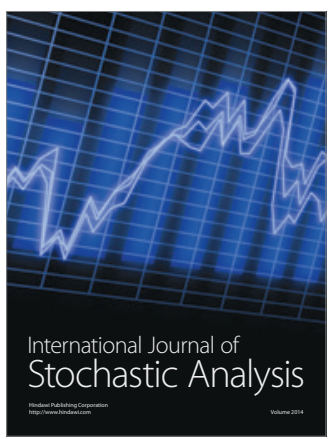

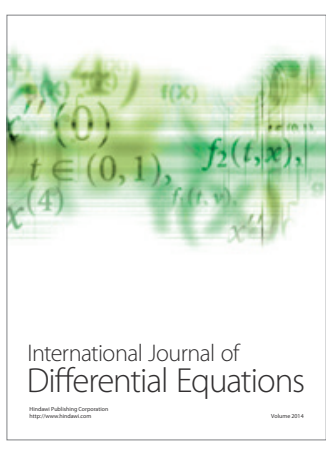
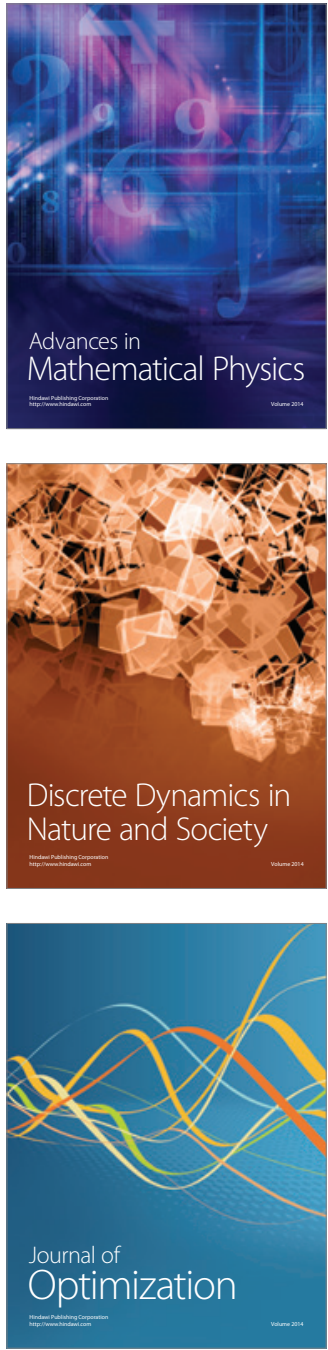